\title{
Emerging Role of Neutrophils in Wound Healing and Tissue Repair: The Routes of Healing
}

\author{
Rajiv Kumar* \\ NIET, National Institute of Medical Science, India \\ *Corresponding author: Rajiv Kumar, NIET, National Institute of Medical Science, India
}

\begin{tabular}{|c|c|}
\hline ARTICLE INFO & ABSTRACT \\
\hline Received: 幽 June 11, 2021 & $\begin{array}{l}\text { Citation: Rajiv Kumar. Emerging Role of Neutrophils in Wound Healing and Tissue } \\
\text { Repair: The Routes of Healing. Biomed J Sci \& Tech Res 36(4)-2021. BISTR. MS.ID.005877. }\end{array}$ \\
\hline Published: 幽 June 17, 2021 & \\
\hline
\end{tabular}

\section{Opinion}

The role of neutrophils in healing, angiogenesis, tissue restoration, controlling host perturbations, and as a bactericidal agent that has an effective antimicrobial activity is important and has therapeutic significance in monitoring the diseased stages and can be examined scientifically with entirely profile. During the microbial invasion, neutrophils mounted up in defense to neutralize its impact. To analyze the contribution of a neutrophil in healing, and how does it perform in different roles in the whole process? Are the key points to be debated [1]. Neutrophils have a superfluity of antimicrobial molecules to end inflammation, which act as indiscriminate killers to eradicate pathogens, clear debris and finally contribute to tissue repair and healing. That is why, the role of neutrophils in tissue damage and repair of cutaneous wounds is important. The unresolved questions interrelated to the intriguing immune cell were countered by underlining the key whys and wherefores of physiology and highlighting recent findings [2]. Neutrophils contributed to normal tissue homeostasis and the process of healing by impelling angiogenesis and cell proliferation. Neutrophil proteases and neutrophil extracellular traps eliminate pathogens and endangering infection. The intricate and delicate chemistry occurred there in the immune system and keratinocytes via wound healing and tissue repair routes, i.e., inflammation, hemostasis, remodeling and proliferation.

The significance of signaling molecules in pro-inflammatory signaling pathway was analyzed during its participation in it, and at the time of activation of myofibroblasts at the time of remodeling [3]. The signaling pathways of wounds, pathological tissue repair, and healing were studied to determine the exact route of the whole process. The key features of the cellular and molecular mechanisms have an important role in controlling inflammation at the event of tissue repair and directed the inflammatory stage for improving the healing and repair response. The important phases of wound healing and tissue repairing is inflammation, proliferation, and remodeling that are considered the most highly complex biological processes (Figure 1). The multifaceted interactions of cytokines, growth factors, chemical mediators and chemokines, transpired in the extracellular matrix in different phases, which normalize the events accompanied by the extracellular molecules, and soluble mediators, transpired in various cells [4]. Further research on "communications of the pathways" will expose the involvement of key chemical inflammatory mediators at the cellular and molecular level [5]. The output of the going on research on extracellular biophysical and biochemical cues notified that modulate distinct intracellular pathways can be modulated, which transpired during tissue restoration.

Ultimately, the dynamic homeostasis existed between cells, and their ECM simultaneously to reestablish subsequent injury. The supremacy of novel technologies to recapitulate the biological mechanisms highlighted by underlining their success in the regeneration, repairing and healing of wounds processes. These success stories are establishing new frontiers in bioengineering [6]. The analysis of interfering processes transpired during healing, and associated disorders persisted in it induce deficiency in tissue repair during chronic inflammation is the key approach, which has many concerns in the interpretation of followed events, to be 
considered immediately for necessary research required in the development of therapy required for a speedy recovery. Herein, author tries to expose the emerging role of neutrophils in wound healing and tissue regeneration and define them as the routes of healing and hope that any further research on the concern needs will be a novel route and new path to achieve the goal of smooth and fast recovery via healing and repair.

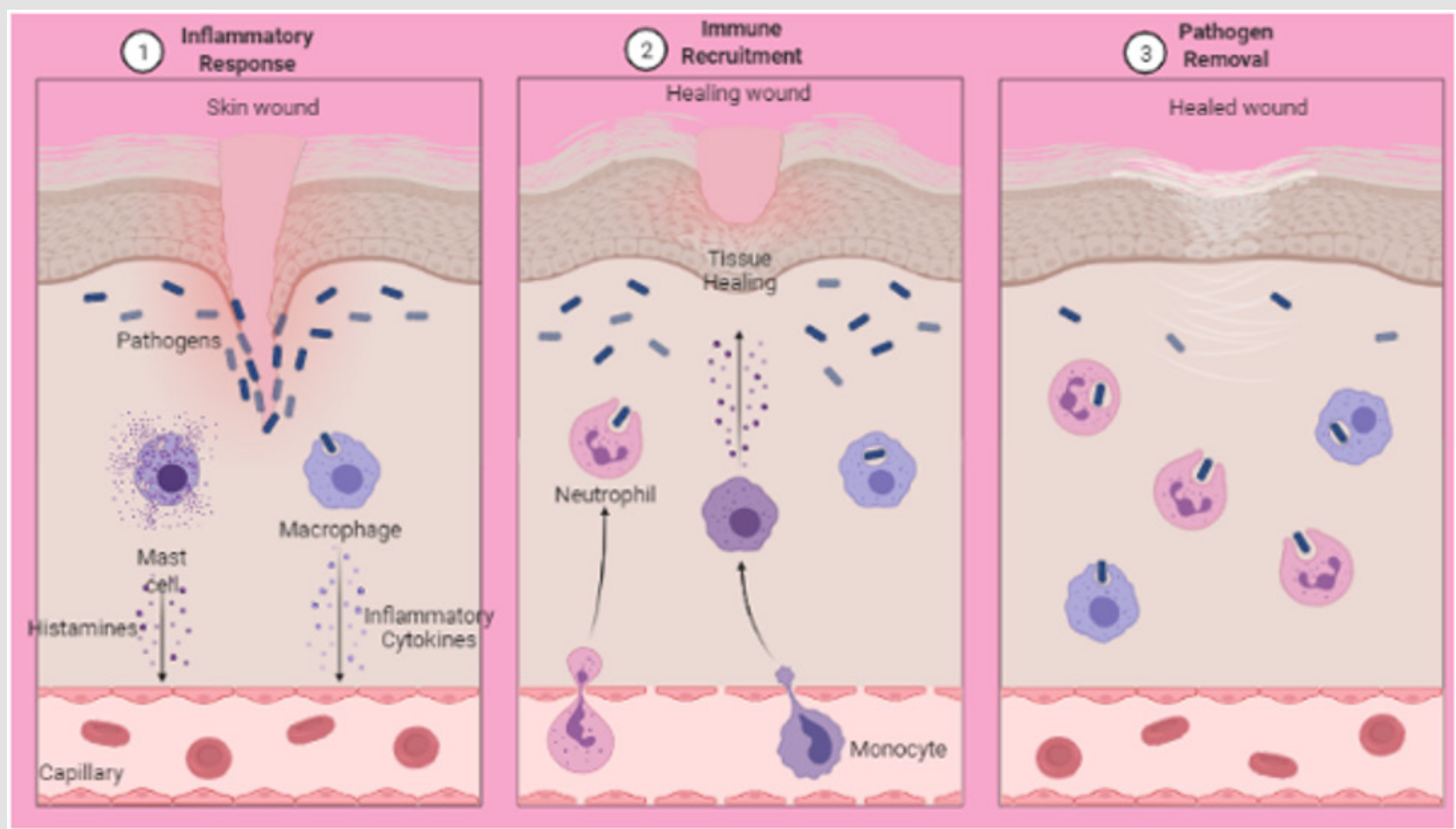

Figure 1: Illustrate the phenomenon of wound healing processes, i.e., hemostasis, inflammation, proliferation, and remodeling. "Adapted and created with permission from [biorender.com] and acknowledged.

\section{References}

1. Houseright RA, Rosowski EE, Lam PY, Mulvaney O, Dewey CN, et al. (2020) Cell type specific gene expression profiling reveals a role for complement component $\mathrm{C} 3$ in neutrophil responses to tissue damage. Sci Rep 10(1): 15716.

2. Kumar R, Gulia K (2021) The convergence of nanotechnology-stem cell, nanotopography-mechanobiology, and biotic-abiotic interfaces: Nanoscale tools for tackling the top killer, arteriosclerosis, strokes, and heart attacks. Nano Sel 2(4): 655-687.

3. Egozi EI, Ferreira AM, Burns AL, Gamelli RL, DiPietro LA (2003) Mast cells modulate the inflammatory but not the proliferative response in healing wounds. Wound Repair Regen 11(1): 46-54.

\section{ISSN: 2574-1241}

DOI: 10.26717/BJSTR.2021.36.005877

Rajiv Kumar. Biomed J Sci \& Tech Res

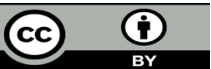

his work is licensed under Creative Commons Attribution 4.0 Licens

Submission Link: https://biomedres.us/submit-manuscript.php
4. Mori R, Power KT, Wang CM, Martin P, Becker DL (2006) Acute downregulation of connexin43 at wound sites leads to a reduced inflammatory reponse, enhanced keratinocyte proliferation and wound fibroblast migration. J Cell Sci 119(Pt 24): 5193-5203.

5. Kumar R, Chhikara BS, Gulia K, Chhillar M (2021) Cleaning the molecular machinery of cellsviaproteostasis, proteolysis and endocytosis selectively, effectively, and precisely: intracellular self-defense and cellular perturbations. Molecular Omics 17: 11-28.

6. De Loura Santana C, Daniela de Fatima Teixeira Silva, Amanda Pires de Souza, Marcos Vinicius Jacinto, Sandra Kalil Bussadori, et al. (2016) Effect of laser therapy on immune cells infiltrate after excisional wounds in diabetic rats. Lasers Surg Med 48(1): 45-51. 